\title{
Enhancing Buckling Limits in Honeycombs for Composite Sandwich Panels.
}

\author{
W. Miller ${ }^{1}$, C.W. Smith ${ }^{1}$, F.L. Scarpa ${ }^{2}$, K.E. Evans ${ }^{1}$. \\ ${ }^{1}$ Engineering Mathematics and Physical Sciences, University of Exeter, EX4 4QF, Exeter, UK. \\ 2 Department of Aerospace Engineering, University of Bristol, BS8 1TR, Bristol, UK.
}

Honeycombs are in wide use as cores in composite sandwich panels, and as such are often subject to combinations of compressive and shear loading. Panel failure is usually defined as either collapse of the honeycomb structure or delamination of the honeycomb and skin components [1]. Such failures are often caused by the collapse of the internal honeycomb structure due to through thickness compression and the subsequent rebounding of the surface skin [1]. It is clear that improving the buckling onset loads of such honeycomb cores can substantially improve performance of some composite sandwich panels. This work presents a study of methods for enhancing the onset buckling load of honeycombs in through thickness compression.

Traditional hexagonal honeycomb cores are compared with a variety of alternative honeycomb geometries, including non-centrosymmetric chiral geometries with various in plane Poisson's ratios (positive and negative), and centrosymmetric negative Poisson's ratio (auxetic) re-entrant geometries. The cylinders and ribs in the chiral honeycombs allows the partial decoupling of the shear stiffness and compressive strength, with ribs providing the shear stiffness and the cylinders the compressive buckling resistance [2].

Auxetic structures are proposed because they display high in-plane shear stiffness and synclastic curvature, they form domes rather than saddle structures, as well as providing improved geometrical characteristics for tailoring to resist compressive buckling and are ideal for consideration as next generation sandwich cores. 


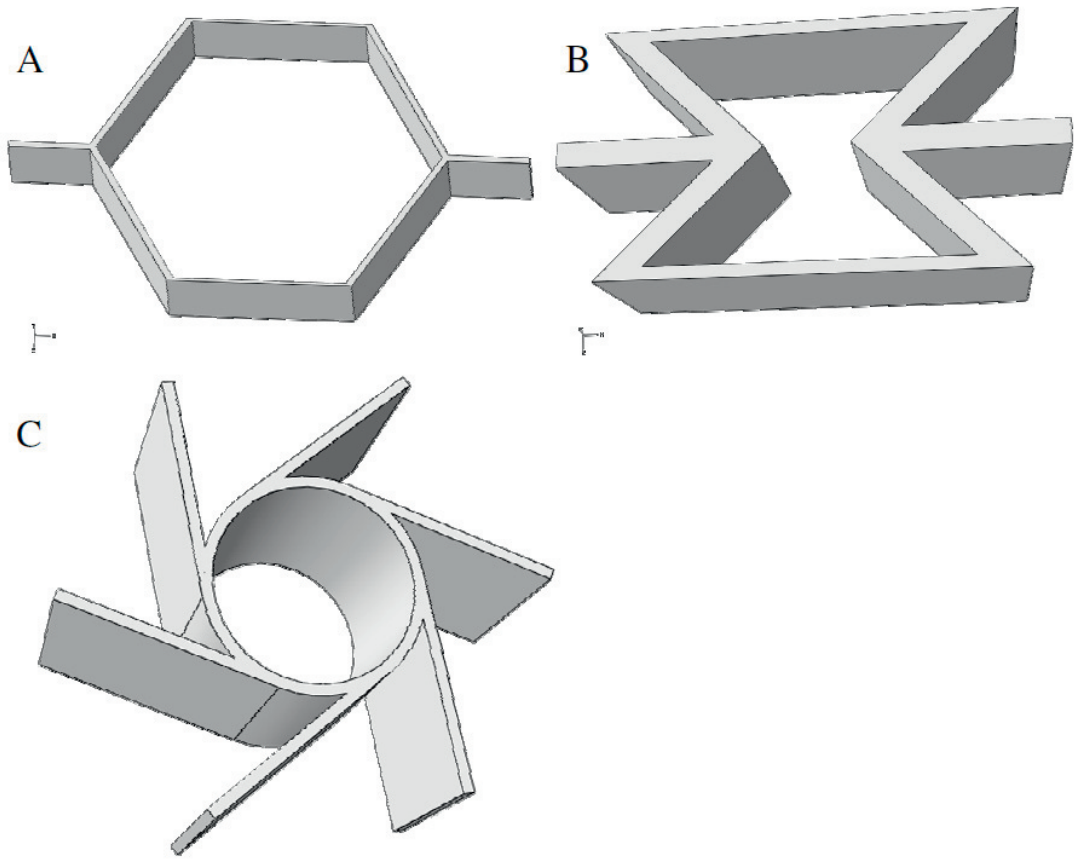

Figure1. A) Conventional hexagonal honeycomb, B) Re-entrant honeycomb structure, C) chiral honeycomb structure.

\section{KEYWORDS:}

Buckling, Negative Poisson's ratio, Chiral, Honeycomb, Multifunctional.

\section{References.}

[1] P.D. Soden. Indentation of composite sandwich beams. The Journal of Strain Analysis for Engineering Design, 1996, 31 (5): 353 - 360.

[2] Scarpa, F, Burriesci, G, Smith FC \& Chambers B. Mechanical and dielectric properties of auxetic honeycomb structures. Aer. J. 2003; 107: 175-183. 